\title{
Erratum to: Long-term forest composition and its drivers in taiga forest in NW Russia
}

\author{
Niina Kuosmanen ${ }^{1}$ Heikki Seppä ${ }^{1}$ Triin Reitalu ${ }^{2}$ Teija Alenius ${ }^{3}$ • \\ Richard H. W. Bradshaw ${ }^{4}$ Jennifer L. Clear ${ }^{5}$. Ludmila Filimonova ${ }^{6}$. \\ Oleg Kuznetsov ${ }^{6} \cdot$ Natalia Zaretskaya $^{7}$
}

Published online: 5 November 2015

(C) Springer-Verlag Berlin Heidelberg 2015

\section{Erratum to: Veget Hist Archaeobot \\ DOI 10.1007/s00334-015-0542-y}

In the original publication of the article, the alignment in Table 1 has been missed inadvertently. The correctly aligned table is found below:

The online version of the original article can be found under doi:10.1007/s00334-015-0542-y.

Niina Kuosmanen

niina.kuosmanen@helsinki.fi

1 Division of Biogeosciences, Department of Geosciences and Geography, University of Helsinki, PO Box 64, 00014 Helsinki, Finland

2 Institute of Geology, Tallinn University of Technology, Ehitajate tee 5, 19086 Tallinn, Estonia

3 Department of Philosophy, History, Culture and Art Studies Archaeology, University of Helsinki, PO Box 59, 00014 Helsinki, Finland

4 Department of Geography and Planning, University of Liverpool, Liverpool L69 7ZT, UK

5 Department of Forest Ecology, Czech University of Life Sciences, 16521 Prague, Czech Republic

6 Karelian Research Centre of RAS, Institute of Biology, 11 Pushkinskaya Street, Petrozavodsk, Karelia 185910, Russia

7 Geological Institute of Russian Academy of Sciences, Moscow, Russia 
Table 1 Variation in individual tree taxa explained by temperature, forest fires, growing site wetness and by joint effect of temperature, forest fires and growing site wetness

\begin{tabular}{|c|c|c|c|c|c|c|c|c|c|c|c|c|c|c|c|}
\hline & \multicolumn{5}{|c|}{ Temperature } & \multicolumn{5}{|c|}{ Forest fires } & \multicolumn{5}{|c|}{ Growing site wetness } \\
\hline & LH & MH & $\mathrm{OH}$ & $\mathrm{OH}^{*}$ & $\mathrm{KH}$ & LH & $\mathrm{MH}$ & $\mathrm{OH}$ & $\mathrm{OH}^{*}$ & $\mathrm{KH}$ & LH & $\mathrm{MH}$ & $\mathrm{OH}$ & $\mathrm{OH}^{*}$ & $\mathrm{KH}$ \\
\hline Picea & 68.3 & 24.3 & 37.8 & 9.0 & 37.6 & $<0$ & - & $<0$ & $<0$ & 2.5 & $<0$ & 13.6 & - & 1.0 & - \\
\hline Pinus & 0.8 & $<0$ & 14.5 & 42.0 & 56.5 & 1.7 & - & $<0$ & $<0$ & $<0$ & 0.6 & $<0$ & - & $<0$ & - \\
\hline Betula & 65.2 & 36.7 & 18.2 & 11.0 & 9.8 & 2.6 & - & $<0$ & $<0$ & 1.4 & 0.3 & 7.7 & - & $<0$ & - \\
\hline Alnus & $<0$ & 46.2 & 41.8 & 2.0 & 18.7 & 3.8 & - & 2.6 & 11.0 & 3.4 & 4.9 & 2.5 & - & 4.0 & - \\
\hline & \multicolumn{5}{|c|}{ Temperature + forest fires } & \multicolumn{5}{|c|}{ Temperature + growing site wetness } & \multicolumn{5}{|c|}{ Forest fires + growing site wetness } \\
\hline Picea & $<0$ & - & 13.7 & $<0$ & $<0$ & $<0$ & 2.0 & - & 0.0 & - & 0.0 & - & - & $<0$ & - \\
\hline Pinus & 2.4 & - & 2.1 & 4.0 & 13.2 & 1.4 & 0.7 & - & $<0$ & - & $<0$ & - & - & 0.0 & - \\
\hline Betula & $<0$ & - & 8.9 & 0.0 & 0.1 & $<0$ & 2.4 & - & 3.0 & - & $<0$ & - & - & 0.0 & - \\
\hline Alnus & $<0$ & - & $<0$ & 7.0 & 1.2 & 0.2 & 0.4 & - & 1.0 & - & 2.0 & - & - & $<0$ & - \\
\hline
\end{tabular}

* Results for Olga Hollow include only 5,000 years and all three variables

- No data 